\title{
Association between day of the week of elective surgery and postoperative mortality
}

\author{
Luc Dubois MD MSc, Kelly Vogt MD MSc, Chris Vinden MD, Jennifer Winick-Ng MSc, J. Andrew McClure MSc, \\ Pavel S. Roshanov MD MSc, Chaim M. Bell MD PhD, Amit X. Garg MD PhD; for the Surgical Investigators Group \\ at ICES Western
}

Cite as: CMAJ 2017 February 27;189:E303-9. doi: 10.1503/cmaj.160511

\begin{abstract}
BACKGROUND: In prior studies, higher mortality was observed among patients who had elective surgery on a Friday rather than earlier in the week. We investigated whether mortality after elective surgery was associated with day of the week of surgery in a Canadian population and whether the association was influenced by surgeon experience and volume.
\end{abstract}

METHODS: We conducted a populationbased retrospective cohort study in the province of Ontario, Canada. We included adults who underwent 1 of 12 elective daytime surgical procedures from Apr. 1,
2002, to Dec. 31, 2012. The primary outcome was 30-day mortality. We used generalized estimating equations to compare outcomes for surgeries performed on different days of the week, adjusting for patient and surgeon factors.

RESULTS: A total of 402899 procedures performed by 1691 surgeons met our inclusion criteria. The median length of hospital stay was 6 (interquartile range $5-8)$ days. Surgeon experience varied significantly by day of week $(p<0.001)$, with surgeons operating on Fridays having the least experience. Nearly all of the patients who had their procedure on a
Friday had postoperative care on the weekend, as compared with $49.1 \%$ of those whose surgery was on a Monday $(p<0.001)$. We found no difference in the 30-day mortality between procedures performed on Fridays and those performed on Mondays (adjusted odds ratio $1.08,95 \%$ confidence interval 0.97-1.21).

INTERPRETATION: Although surgeon experience differed across days of the week, the risk of 30-day mortality after elective surgery was similar regardless of which day of the week the procedure took place. n 3 prior studies, a higher risk of death was observed among patients who had elective surgery on a Friday than earlier in the week. ${ }^{1-3}$ This "weekday effect" was most pronounced in a UK study, which reported a $44 \%$ increase in the risk of death among patients whose surgery took place on a Friday compared with Monday. ${ }^{1}$ Yet the weekday effect is still a matter of debate, as are the possible mechanisms to explain it., It may be that senior surgeons prefer to operate earlier in the week and tend to have better outcomes over a wide range of surgical procedures than less experienced surgeons. ${ }^{5}$ Friday surgeries also result in a greater proportion of postoperative care occurring on the weekend, a time when there are fewer health care personnel in the hospital and less access to diagnostic services. ${ }^{6-12}$

We investigated whether elective surgery performed later in the week was associated with a higher mortality than elective surgery performed earlier in the week in a Canadian population and whether the association was influenced by surgeon experience and volume.

\section{Methods}

\section{Study setting and design}

Residents of the province of Ontario (2012 population 13.4 million ${ }^{13}$ ) have universal access to hospital care and physician services, and all eligible health care encounters are recorded in administrative databases. Relevant data sets were linked using unique, encoded identifiers and analyzed at the Institute for Clinical Evaluative Sciences (ICES) Western site. The reporting of this study followed the REporting of studies Conducted using Observational Routinely-collected health Data (RECORD) statement (Table S1 in Appendix 1, available at www.cmaj.ca/lookup/suppl/ doi:10.1503/cmaj.160511/-/DC1). ${ }^{14}$

In this retrospective cohort study, we included all adult residents of Ontario who underwent 1 of 12 elective procedures from Apr. 1, 2002, to Dec. 31, 2012: esophagectomy or gastrectomy; pancreaticoduodenectomy; nephrectomy; cystectomy; partial liver resection; lobectomy or pneumonectomy; colorectal resec- 
tion; total hip or knee replacement; coronary artery bypass graft surgery or aortic valve replacement; aortic aneurysm repair; lower limb revascularization; and carotid endarterectomy. (Procedure codes are available in Table S2 of Appendix 1.) We chose procedures that are commonly done only electively and typically result in at least a 2-day hospital stay. We did not include outpatient procedures because they have limited in-hospital postoperative care and generally have lower mortality.

We excluded patients if their procedure was performed outside of regular office hours (evening, weekend or holiday) or if they underwent more than 1 of the 12 index procedures on the same date (Table S3 in Appendix 1). For patients who underwent multiple procedures during the study period, we included only the first procedure to retain a per-patient analysis.

\section{Data sources}

We obtained data on surgeon and patient characteristics from 5 linked health care administrative databases. Diagnostic, procedural and clinical patient data are recorded in the Canadian Institute for Health Information Discharge Abstract Database and Same Day Surgery databases, whereas health claims are recorded in the Ontario Health Insurance Plan (OHIP) database. The Registered Persons Database, which contains vital statistics for all residents of Ontario, was used to obtain patient demographic data, including age, sex and date of death. Physician demographic data, including age, years of practice and location of medical training, were retrieved from the ICES Physician Database. We determined hospital teaching status from a list of academic hospitals issued by Health Force Ontario. ${ }^{15}$

\section{Outcome measures}

The primary outcome was all-cause mortality within 30 days after the procedure date. Secondary outcomes included 2-day, 90-day and in-hospital all-cause mortality; length of hospital stay; admission to an intensive care unit (ICU) after the procedure; hospital readmission within 30 days after the procedure; and reoperation within 30 days after the procedure. Mortality data were obtained from the Registered Persons Database, OHIP billing records were used to identify reoperations, and the Discharge Abstract Database was used to capture all other outcomes.

\section{Covariates}

Patient-related variables included age, sex, expected resource utilization and socioeconomic status (approximated using the patients' neighbourhood income quintile). We determined patients' expected resource utilization using Resource Utilization Bands (RUBs), a component of the Johns Hopkins Adjusted Clinical Groups case-mix system. ${ }^{16}$ We categorized the expected resource utilization as low (RUB groups 0-3), moderate (RUB 4) or high (RUB 5). The Adjusted Clinical Groups system has been shown to predict mortality accurately using administrative data. ${ }^{17}$ Each patient-related variable was included because of its potential impact on delay of intervention and surgical outcomes.

To account for potential differences in the effect of day of the week among various surgical procedures and among different practice settings, we included type of procedure, year of proce- dure and institution teaching status into our model. Given the impact of surgeon expertise on outcomes, we also recorded surgeons' years of experience (estimated as years since medical school graduation, less 5 years to allow for residency) and annual procedure-specific volume (for that specific procedure, the total number performed during the fiscal year in question).

\section{Statistical analyses}

We assessed differences in patient-, surgeon- and procedurerelated characteristics by day of the week in a univariable analysis using analysis of variance for continuous variables, the $\chi^{2}$ test for categorical variables and the nonparametric Kruskal-Wallis test for non-normal continuous variables.

We conducted multivariable analysis using multiple logistic regression to model the association between day of week and each of the primary and secondary outcomes (other than length of hospital stay). To account for the clustering effect of patients within surgeons and hospitals, we used generalized estimating equations with an exchangeable working correlation matrix. ${ }^{18}$ For missing data, we used case-wise deletion. Data were missing only for the income quintile variable $(n=1293[0.003 \%])$; this variable was missing owing to incomplete administrative records and was likely missing completely at random.

We used restricted cubic splines with 5 knots to test the assumption of linearity for each of the continuous covariates. ${ }^{19,20} \mathrm{Al}$ though evidence of nonlinearity was observed, model estimates of odds ratios (ORs) where linearity was assumed were within 0.01 of those produced when restricted cubic splines were used. Given the similarity of results between the 2 approaches, restricted cubic splines were abandoned in favour of model simplicity and ease of interpretation.

In the primary analysis, day of week was modelled as a categorical variable, with Monday as the reference group, an approach similar to that described by Aylin and colleagues. ${ }^{1}$ In sensitivity analyses, we investigated the impact of varying the day of week as the reference category. In the first sensitivity analysis, we collapsed Monday, Tuesday and Wednesday into a single reference group and compared it with each of Thursday and Friday. In a second post hoc sensitivity analysis, Monday and Friday were collapsed and compared with the combined reference category of Tuesday through Thursday. All of the primary and sensitivity analyses were performed with data for the entire study population.

We performed subgroup analyses (planned a priori) to determine whether the association between the day of week and the outcomes was modified by the specific procedure performed or the procedure risk. We defined high-risk procedures as those with a greater than $2 \%$ risk of death within 30 days, as observed in the current study. We performed an additional subgroup analysis (planned post hoc) that was restricted to patients with a low level of complexity (RUB groups $0-3$ ). The purpose of this analysis was to assess the potential impact of differing levels of complexity across days of the week as well as the potential impact of referring more challenging patients to more senior surgeons. We also conducted an unadjusted analysis examining the association between surgeons' years of experience and 30-day mortality. We examined differences in the proportion of patients for 
whom part of their postoperative care occurred over the weekend by day of surgery using a Cochran-Armitage test for trend. Odds ratios can be interpreted as relative risks (appropriate given the incidences observed).

All analyses were conducted with the use of SAS version 9.3 (PROC GENMOD; SAS Institute).

\section{Ethics approval}

The study was approved by the Sunnybrook Health Sciences Centre Research Ethics Board.

\section{Results}

\section{Patient and surgeon characteristics}

A total of 402899 procedures (50.6\% in men) performed by 1691 different surgeons met our inclusion criteria. The median length of hospital stay after surgery was 6 (interquartile range 5-8) days. The greatest number of surgeries was performed on Tuesdays ( $n=$
95654) and the least on Fridays ( $n=65139)$. Patient age, sex and expected resource utilization category varied little by day of the week (Table 1). Hip and knee replacements accounted for more than half (57.7\%) of the procedures, colorectal resection for $11.6 \%$ and coronary artery bypass graft surgery or aortic valve replacement for $11.2 \%$.

Surgeon experience differed significantly by the day of week $(p<0.001)$. Experience was highest on Tuesdays and Wednesdays (on average $19 \mathrm{yr}$ in practice) and lowest on Mondays and Fridays (on average $16 \mathrm{yr}$ in practice). The proportion of surgeons who were operating in their first 5 years of practice was nearly twice as high on Fridays as on Tuesdays ( $11.1 \%$ v. $5.7 \%$; Figure 1 ). The median annual procedure volume also differed significantly by the day of week $(p<0.001)$ and was highest for surgeons who operated on Tuesdays (80 cases) and lowest on Fridays (72 cases) (Table 1). Unadjusted analysis of the effect of surgeon experience on 30-day mortality did not show a significant association (OR 1.00, 95\% confidence interval [CI] 1.00-1.01).

Table 1: Baseline characteristics of patients, procedures and surgeons for all procedures combined, by day of the week of surgery

\begin{tabular}{|c|c|c|c|c|c|}
\hline \multirow[b]{2}{*}{ Characteristic } & \multicolumn{5}{|c|}{ Day of surgery; no. $(\%)^{\star}$} \\
\hline & $\begin{array}{c}\text { Monday } \\
(n=77082)\end{array}$ & $\begin{array}{c}\text { Tuesday } \\
(n=95654)\end{array}$ & $\begin{array}{l}\text { Wednesday } \\
(n=86853)\end{array}$ & $\begin{array}{l}\text { Thursday } \\
(n=78 \text { 171) }\end{array}$ & $\begin{array}{c}\text { Friday } \\
(n=65 \text { 139) }\end{array}$ \\
\hline \multicolumn{6}{|l|}{ Patient } \\
\hline Patient age, yr, mean \pm SD & $66.5 \pm 11.3$ & $66.2 \pm 11.6$ & $66.5 \pm 11.5$ & $66.2 \pm 11.6$ & $66.4 \pm 11.7$ \\
\hline Patient sex, female & $38281(49.7)$ & $47209(49.4)$ & $42609(49.1)$ & $38639(49.4)$ & $32405(49.7)$ \\
\hline \multicolumn{6}{|l|}{ Expected resource utilization } \\
\hline Low (RUB 0-3) & $36961(48.0)$ & $45297(47.4)$ & $40400(46.5)$ & 36844 (47.1) & $30150(46.3)$ \\
\hline Moderate (RUB 4) & $23983(31.1)$ & $30060(31.4)$ & $27315(31.4)$ & $24598(31.5)$ & $20615(31.6)$ \\
\hline High (RUB 5) & $16138(20.9)$ & $20297(21.2)$ & $19138(22.0)$ & $16729(21.4)$ & $14374(22.1)$ \\
\hline \multicolumn{6}{|l|}{ Neighbourhood income quintile $†$} \\
\hline 1 (lowest) & $13721(17.8)$ & $16909(17.7)$ & $15939(18.4)$ & $14107(18.0)$ & $11783(18.1)$ \\
\hline 2 & $15581(20.2)$ & $19477(20.4)$ & $17845(20.5)$ & $15678(20.1)$ & $13166(20.2)$ \\
\hline 3 & $15287(19.8)$ & $19015(19.9)$ & $17275(19.9)$ & $15543(19.9)$ & $12962(19.9)$ \\
\hline 4 & $15911(20.6)$ & $19533(20.4)$ & $17405(20.0)$ & $16160(20.7)$ & $13376(20.5)$ \\
\hline 5 (highest) & $16350(21.2)$ & $20416(21.3)$ & $18121(20.9)$ & $16438(21.0)$ & $13608(20.9)$ \\
\hline \multicolumn{6}{|l|}{ Surgeon/procedure $\ddagger$} \\
\hline Teaching hospital & $27542(35.7)$ & $35069(36.7)$ & $30788(35.4)$ & $30051(38.4)$ & $25026(38.4)$ \\
\hline Assistant present & $54061(70.1)$ & $66302(69.3)$ & $61357(70.6)$ & $53792(68.8)$ & $43799(67.2)$ \\
\hline Physician age, yr, mean \pm SD & $47.5 \pm 9.0$ & $49.2 \pm 9.1$ & $48.8 \pm 9.1$ & $48.9 \pm 9.3$ & $47.1 \pm 9.6$ \\
\hline Physician sex, female & $4427(5.7)$ & $3268(3.4)$ & $4433(5.1)$ & $3360(4.3)$ & $3021(4.6)$ \\
\hline Years of practice, median (IQR) & $16(9-24)$ & $19(12-26)$ & $19(11-25)$ & $18(11-26)$ & $16(8-24)$ \\
\hline Non-Canadian medical graduate & $12664(16.4)$ & $13038(13.6)$ & $16163(18.6)$ & $15032(19.2)$ & $10916(16.8)$ \\
\hline Annual procedure volume,§ median (IQR) & $76(31-120)$ & $80(32-125)$ & $77(29-117)$ & $73(27-116)$ & $72(27-118)$ \\
\hline
\end{tabular}




\section{Effect on mortality and other outcomes}

The overall 30 -day mortality was $0.84 \%$. There was no consistent association between the day of week of the surgery and 30-day mortality in the unadjusted and adjusted analyses for the overall cohort when Friday was compared with Monday (adjusted OR 1.08, $95 \% \mathrm{Cl} 0.97-1.21$ ) (Table 2). Similarly, the risk of any of the secondary outcomes was not consistently higher on Friday than on other days of the week (Table 3). There was no difference in the adjusted odds of 30-day readmission (adjusted OR 1.02, 95\% Cl 0.98-1.06) or 30-day reoperation (adjusted OR $0.96,95 \% \mathrm{Cl} 0.90-1.02$ ) when Fri-

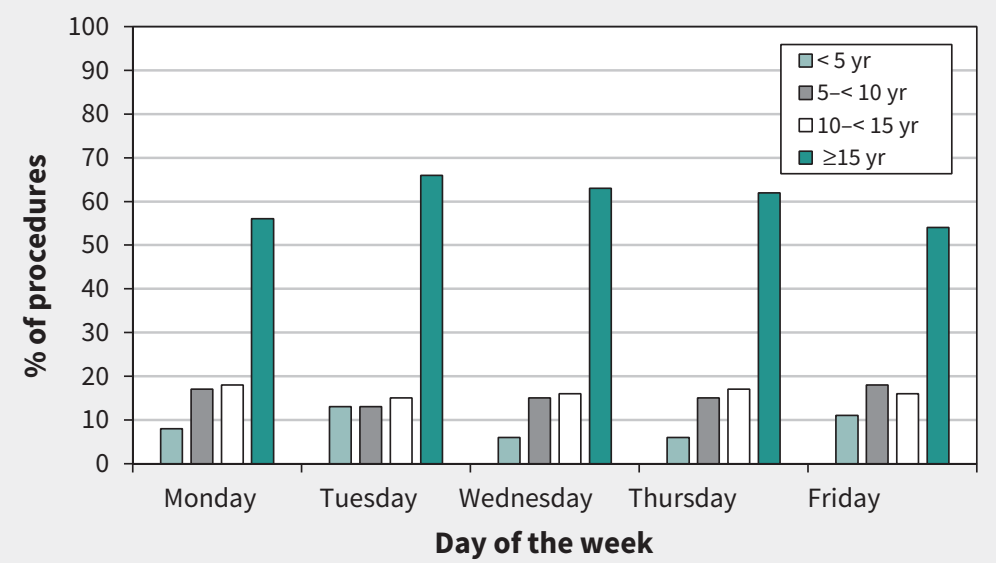

Figure 1: Proportion of surgical procedures performed according to years of surgeon experience, by day of the week. day was compared with Monday. The risk of ICU admission was slightly higher on Friday than on Monday (adjusted OR 1.07, 95\% Cl 1.02-1.12). The median length of hospital stay was shorter for patients whose surgery was performed on a Monday than for those whose surgery was on another day of the week ( $5 \mathrm{v} .6 \mathrm{~d}$ ).

In subgroup analyses in which we examined each type of procedure separately, we found no consistent association between the day of the week of surgery and 30-day mortality across multiple types of procedures (Table S4 in Appendix 1). Similarly, there was no association between the day of week and 30-day mortality when the procedures were classified as low risk or high risk (Table S5 in Appendix 1). The results for the cohort restricted to patients with a low level of complexity (RUB groups 0-3) were consistent with results from the primary analysis (data not shown).

Changing the categorization of day of week to (a) Monday through Wednesday versus either Thursday or Friday or (b) combining Monday and Friday and comparing them with to the remaining 3 days of the week combined showed no difference in the association with 30-day mortality (Table S6 in Appendix 1).

\section{Interpretation}

More than $\mathbf{3 0 0}$ million elective surgeries are performed worldwide each year. ${ }^{1,21}$ If it is true that a person's risk of death is higher if their elective

Table 2: Mortality outcomes for all procedures combined, by day of the week of surgery

\begin{tabular}{|c|c|c|c|c|c|c|}
\hline Outcome & $\begin{array}{c}\text { Monday } \\
(n=77082)\end{array}$ & $\begin{array}{c}\text { Tuesday } \\
(n=95654)\end{array}$ & $\begin{array}{l}\text { Wednesday } \\
(n=86853)\end{array}$ & $\begin{array}{l}\text { Thursday } \\
(n=78 \text { 171) }\end{array}$ & $\begin{array}{c}\text { Friday } \\
(n=65 \text { 139) }\end{array}$ & $p$ value ${ }^{\star}$ \\
\hline \multicolumn{7}{|l|}{ 30-day mortality } \\
\hline No. (\%) of events & $622(0.8)$ & $809(0.8)$ & $719(0.8)$ & $646(0.8)$ & $595(0.9)$ & - \\
\hline Unadjusted OR (95\% CI) & 1.00 (ref) & $1.05(0.94-1.16)$ & $1.03(0.92-1.14)$ & $1.02(0.92-1.14)$ & $1.13(1.01-1.27)$ & 0.2 \\
\hline Adjusted OR† (95\% CI) & 1.00 (ref) & $1.02(0.91-1.14)$ & $0.97(0.87-1.08)$ & $1.01(0.90-1.13)$ & $1.08(0.97-1.21)$ & 0.4 \\
\hline \multicolumn{7}{|l|}{ 2-day mortality } \\
\hline No. (\%) of events & $112(0.1)$ & $126(0.1)$ & $112(0.1)$ & $107(0.1)$ & $85(0.1)$ & - \\
\hline Unadjusted OR (95\% Cl) & 1.00 (ref) & $0.91(0.70-1.17)$ & $0.89(0.68-1.15)$ & $0.94(0.72-1.23)$ & $0.90(0.68-1.19)$ & 0.9 \\
\hline Adjusted OR† (95\% CI) & 1.00 (ref) & $0.90(0.69-1.17)$ & $0.85(0.66-1.09)$ & $0.93(0.71-1.20)$ & $0.87(0.65-1.16)$ & 0.8 \\
\hline \multicolumn{7}{|l|}{ In-hospital mortality } \\
\hline No. (\%) of events & $516(0.7)$ & $679(0.7)$ & $597(0.7)$ & $540(0.7)$ & $482(0.7)$ & - \\
\hline Unadjusted OR (95\% CI) & 1.00 (ref) & $1.06(0.95-1.19)$ & $1.03(0.91-1.16)$ & $1.03(0.91-1.16)$ & $1.11(0.98-1.25)$ & 0.6 \\
\hline Adjusted OR† (95\% CI) & 1.00 (ref) & $1.02(0.91-1.16)$ & $0.97(0.86-1.09)$ & $1.02(0.90-1.15)$ & $1.05(0.93-1.19)$ & 0.8 \\
\hline \multicolumn{7}{|l|}{ 90-day mortality } \\
\hline No. (\%) of events & $1075(1.4)$ & $1387(1.5)$ & $1235(1.4)$ & $1151(1.5)$ & $1027(1.6)$ & - \\
\hline Unadjusted OR (95\% CI) & 1.00 (ref) & $1.04(0.96-1.13)$ & $1.02(0.94-1.11)$ & $1.06(0.97-1.15)$ & $1.13(1.04-1.23)$ & 0.050 \\
\hline Adjusted OR† (95\% Cl) & 1.00 (ref) & $1.02(0.93-1.12)$ & $0.97(0.89-1.05)$ & $1.05(0.96-1.15)$ & $1.09(1.00-1.19)$ & 0.07 \\
\hline
\end{tabular}


surgery is on a Friday compared with earlier in the week, this would have important implications for informed consent and public health. We hypothesized that less experienced surgeons would operate on a Friday compared with other days of the week. Our study showed this to be true, perhaps because senior surgeons prefer to operate mid-week in an effort to limit their clinical obligations related to postoperative care on the weekend. Reassuringly, we found no association between day of the week of surgery and the outcomes of elective surgery. This lack of association was observed in the unadjusted and adjusted analyses in the entire study cohort, and in subgroups defined by the type of surgery and the risk of surgery.

Our findings are in contrast to those of Aylin and colleagues ${ }^{1}$ in their analysis of UK data showing a $44 \%$ increase in 30-day mortality following elective surgery occurring on Friday when compared with Monday. This discrepancy is even more striking when you consider that their study also included lower risk groups, and it had a crude rate of death similar to the rate in our study (6.7 per 1000 and 8.4 per 1000, respectively). In a follow-up paper, their group also showed that less experienced consultants operate on Fridays compared with earlier in the week; however, the difference was less marked (7.9 v. 8.5 median years of experience) than in our study, and differences in consultant seniority did not have an impact on either mortality or the association between the day of the week and mortality. ${ }^{22}$ Our results in combination with those of Aylin's group point to other causes for the weekday effect than variations in surgeon experience.
A global comparative study examining variation in mortality following elective surgery showed that the presence of a weekday effect was not consistent across the countries studied (England, Australia, United States and the Netherlands). ${ }^{3}$ The Netherlands showed a strong weekday effect, whereas Australia showed little effect. In combination with our results, these findings suggest that variation in mortality following elective surgery over the course of the week likely represents differences in process of care and other organizational factors that differ between health care systems, such as hand-off procedures for patient care, staffing levels, availability of consulting services or diagnostic studies, access to specialized treatments and clinical acuity (worsening symptoms, impending complications). ${ }^{1,3,23}$

Although many studies have identified a weekday or weekend effect, few have attempted to discern the mechanisms behind it. Patients who have elective surgery on a Friday have a larger proportion of their postoperative care occurring on weekends, compared with patients who have their procedure earlier in the week. Previous studies investigating the effect of hospital staffing levels have shown that hospitals with lower levels of staffing on the weekend have higher rates of death following emergent weekend admissions. ${ }^{12,24,25}$ Reduced weekend availability of interventional therapies, such as endoscopies for urgent bleeding, cardiac catheterizations for myocardial infarctions and thrombolysis for stroke, has also been found to be associated with increased mortality. ${ }^{6,7,26-29}$ Variability in the availability of diagnostic and therapeutic services during weekends and variations in hospital staff-

Table 3: Health services outcomes for all procedures combined, by day of the week of surgery

\begin{tabular}{|c|c|c|c|c|c|c|}
\hline Outcome & $\begin{array}{c}\text { Monday } \\
(n=77082)\end{array}$ & $\begin{array}{l}\text { Tuesday } \\
(n=95654)\end{array}$ & $\begin{array}{l}\text { Wednesday } \\
(n=86853)\end{array}$ & $\begin{array}{l}\text { Thursday } \\
(n=78 \text { 171) }\end{array}$ & $\begin{array}{c}\text { Friday } \\
(n=65 \text { 139) }\end{array}$ & $p$ value* \\
\hline \multicolumn{7}{|l|}{ 30-day readmission } \\
\hline No. (\%) of events & $6100(7.9)$ & $7574(7.9)$ & $7057(8.1)$ & $6338(8.1)$ & $5169(7.9)$ & - \\
\hline Unadjusted OR (95\% CI) & 1.00 (ref) & $1.00(0.97-1.04)$ & $1.03(0.99-1.07)$ & $1.03(0.99-1.07)$ & $1.00(0.96-1.04)$ & 0.3 \\
\hline Adjusted OR† $(95 \% \mathrm{CI})$ & 1.00 (ref) & $1.05(1.01-1.09)$ & $1.06(1.01-1.10)$ & $1.04(1.00-1.08)$ & $1.02(0.98-1.06)$ & 0.06 \\
\hline \multicolumn{7}{|l|}{ 30-day reoperation } \\
\hline No. (\%) of events & $3219(4.2)$ & $3943(4.1)$ & $3870(4.5)$ & $3447(4.4)$ & $2743(4.2)$ & - \\
\hline Unadjusted OR (95\% CI) & 1.00 (ref) & $0.99(0.94-1.03)$ & $1.07(1.02-1.12)$ & $1.06(1.01-1.11)$ & $1.01(0.96-1.06)$ & 0.001 \\
\hline Adjusted OR† (95\% CI) & 1.00 (ref) & $0.97(0.92-1.03)$ & $1.03(0.97-1.09)$ & $1.01(0.95-1.07)$ & $0.96(0.90-1.02)$ & 0.2 \\
\hline \multicolumn{7}{|l|}{ ICU admission } \\
\hline No. (\%) of events & $19193(24.9)$ & $23329(24.4)$ & $22392(25.8)$ & $18579(23.8)$ & $15358(23.6)$ & - \\
\hline Unadjusted OR (95\% CI) & 1.00 (ref) & $0.97(0.95-0.99)$ & $1.05(1.02-1.07)$ & $0.94(0.92-0.96)$ & $0.93(0.91-0.95)$ & $<0.001$ \\
\hline Adjusted OR† (95\% CI) & 1.00 (ref) & $1.05(1.00-1.10)$ & $1.02(0.98-1.07)$ & $1.09(1.03-1.15)$ & $1.07(1.02-1.12)$ & 0.03 \\
\hline \multicolumn{7}{|l|}{ Length of stay, $d$} \\
\hline Median (IQR) & $5(4-8)$ & $6(4-8)$ & $6(5-8)$ & $6(5-8)$ & $6(5-8)$ & $<0.001$ \\
\hline Mean \pm SD & $7.5 \pm 9.1$ & $7.6 \pm 9.2$ & $7.7 \pm 8.8$ & $7.8 \pm 9.0$ & $7.9 \pm 8.8$ & $<0.001$ \\
\hline Weekend stay, no. (\%)‡ & $37828(49.1)$ & $68699(71.8)$ & $79144(91.1)$ & 76897 (98.4) & 65076 (99.9) & $<0.001$ \\
\hline \multicolumn{7}{|c|}{$\begin{array}{l}\text { Note: } \mathrm{Cl}=\text { confidence interval, } \mathrm{ICU}=\text { intensive care unit, } \mathrm{QQR}=\text { interquartile range, } \mathrm{OR}=\text { odds ratio, ref = reference category, } \mathrm{SD}=\text { standard deviation. } \\
{ }^{*} p \text { values represent an overall test of significance, not a comparison of individual odds ratios. } \\
\text { tAdjusted for patient age, sex, comorbidity (RUB), income quintile, hospital teaching status, procedure, year of the procedure, surgeon years of experience, and surgeon procedure- } \\
\text { specific annual volume. } \\
\text { tWeekend stay represents the number (\%) of patients whose hospital stay included Saturday, Sunday or both. }\end{array}$} \\
\hline
\end{tabular}


ing levels across different health care systems likely explain the inconsistency of the weekday effect.

We chose not to include patients undergoing surgery on weekends in our analysis. In Canada, surgical procedures that occur on the weekend likely represent an emergent or semi-urgent clinical situation (e.g., colon cancer with bowel obstruction or symptomatic aortic aneurysm), which would carry an increased risk of adverse events when compared with truly elective cases. This notion is supported by Mclsaac and colleagues, ${ }^{23}$ who found that patients within a Canadian population who had "elective" surgery on the weekend differed systematically from those whose elective surgery was on a weekday.

\section{Limitations}

Our study has several limitations associated with the use of administrative data. We linked the surgeons to procedures via billing records but did not account for the potential influence of trainees. However, we did adjust for institution teaching status.

Missing data are a concern for all research based on administrative data. For our study, it seems unlikely that data would be missing differentially by day of the week of surgery; therefore, we did not expect a meaningful bias due to missing data.

Although we adjusted for several patient and surgeon factors, the databases did not contain variables such as laboratory values, symptomatic status or specific indications for surgery. Consequently, our ability to capture patients' baseline level of risk accurately was limited and may have resulted in residual confounding. However, concern regarding this issue should be mitigated by results from the subgroup analysis restricted to patients with a low level of complexity, which were similar to those obtained for the full cohort.

Despite these limitations, the accuracy of administrative data to predict mortality has been shown to approach that of clinical databases. ${ }^{30}$ Although we did not observe a weekday effect in our study, inclusion of different procedures or a larger sample might have yielded a significant difference in surgical outcomes by the day of the week of surgery. Nevertheless, given the size of our study and the relatively low observed point estimates, it seems unlikely that we failed to detect any clinically meaningful associations.

\section{Conclusion}

Our study showed that the risk of 30-day mortality after elective surgery was similar regardless of which day of the week the procedure took place. The results should be reassuring to administrators, policy-makers and the public in Ontario. We did find that less experienced surgeons and those with slightly lower annual procedure volumes tended to operate more frequently on Mondays and Fridays, whereas more senior surgeons tended to operate mid-week. Regardless, these variations in surgeon experience and volume did not appear to contribute to any potential weekday effect on mortality. That our findings differ from those of previous studies suggests that the weekday effect is not a universal phenomenon across all health care systems and that the factors responsible for this effect are likely correctable in jurisdictions where it occurs. Future studies aimed at discerning the mecha- nisms behind the weekday effect should examine differences in processes of care and other organizational factors across health care systems.

\section{References}

1. Aylin P, Alexandrescu R, Jen MH, et al. Day of week of procedure and 30 day mortality for elective surgery: retrospective analysis of hospital episode statistics. BMJ 2013;346:f2424.

2. Zare MM, Itani KM, Schifftner TL, et al. Mortality after nonemergent major surgery performed on Friday versus Monday through Wednesday. Ann Surg 2007;246:866-74.

3. Ruiz M, Bottle A, Aylin PP. The Global Comparators project: international comparison of 30-day in-hospital mortality by day of the week. BMJ Qual Saf 2015;24:492-504.

4. Lilford RJ, Chen YF. The ubiquitous weekend effect: moving past proving it exists to clarifying what causes it. BMJ Qual Saf 2015;24:480-2.

5. Maruthappu M, Gilbert BJ, El-Harasis MA, et al. The influence of volume and experience on individual surgical performance: a systematic review. Ann Surg 2015;261:642-7.

6. Bell CM, Redelmeier DA. Waiting for urgent procedures on the weekend among emergently hospitalized patients. Am J Med 2004;117:175-81.

7. Palmer WL, Bottle A, Davie C, et al. Dying for the weekend: a retrospective cohort study on the association between day of hospital presentation and the quality and safety of stroke care. Arch Neurol 2012;69:1296-302.

8. Needleman J, Buerhaus P, Pankratz VS, et al. Nurse staffing and inpatient hospital mortality. N Engl J Med 2011;364:1037-45.

9. Blecker S, Austrian JS, Shine D, et al. Monitoring the pulse of hospital activity: electronic health record utilization as a measure of care intensity. $J$ Hosp Med 2013;8:513-8.

10. Concha OP, Gallego B, Hillman K, et al. Do variations in hospital mortality patterns after weekend admission reflect reduced quality of care or different patient cohorts? A population-based study. BMJ Qual Saf 2014;23:215-22.

11. Weekend admissions and in-hospital mortality. Ottawa: Canadian Institute for Health Information (CIHI); 2014.

12. Goddard AF, Lees P. Higher senior staffing levels at weekends and reduced mortality. BMJ 2012;344:e67.

13. Table 051-0001: Estimates of population, by age group and sex for July 1, Canada, provinces and territories [annual]. Ottawa: Statistics Canada. Available: http://www5.statcan.gc.ca/cansim/a26?lang=eng\&id=510001 [select data for Ontario] (accessed 2014 Nov. 27).

14. Benchimol El, Smeeth L, Guttmann A, et al. The REporting of studies Conducted using Observational Routinely-collected health Data (RECORD) Statement. PLoS Med 2015;12:e1001885.

15. Ontario academic health sciences centres [Appendix A]. Toronto: HealthForceOntario. Available: www.healthforceontario.ca/en/M4/Clerkship_Travel_Program/ Program_Guidelines/Ontario_Academic_Health_Science_Centres (accessed 2014 Aug. 6).

16. The John Hopkins ACG Case-Mix System, version 6.0 release notes. Baltimore (MD): The Johns Hopkins University Bloomberg School of Public Health; 2003.

17. Austin PC, van Walraven C, Wodchis WP, et al. Using the Johns Hopkins Aggregated Diagnosis Groups (ADGs) to predict mortality in a general adult population cohort in Ontario, Canada. Med Care 2011;49:932-9.

18. Hubbard AE, Ahern J, Fleischer NL, et al. To GEE or not to GEE: comparing population average and mixed models for estimating the associations between neighborhood risk factors and health. Epidemiology 2010;21:467-74.

19. Croxford R. Restricted cubic spline regression: a brief introduction. Toronto: Institute for Clinical Evaluative Sciences. Available: http://support.sas.com/resources/ papers/proceedings16/5621-216.pdf (accessed 2016 Jun. 2).

20. Harrell FE. Regression modeling strategies with applications to linear models, logistic regression, and survival analysis. New York: Springer-Verlag New York; 2010.

21. Weiser TG, Haynes AB, Molina G, et al. Estimate of the global volume of surgery in 2012: an assessment supporting improved health outcomes. Lancet 2015; 385(Suppl 2):S11.

22. Ruiz M, Bottle A, Aylin PP. Exploring the impact of consultants' experience on hospital mortality by day of the week: a retrospective analysis of hospital episode statistics. BMJ Qual Saf 2016;25:337-44.

23. Mclsaac DI, Bryson GL, van Walraven C. Elective, major noncardiac surgery on the weekend: a population-based cohort study of 30-day mortality. Med Care 2014;52:557-64.

24. Ozdemir BA, Sinha S, Karthikesalingam A, et al. Mortality of emergency general surgical patients and associations with hospital structures and processes. $\mathrm{Br} J$ Anaesth 2016;116:54-62. 
25. Ricciardi R, Nelson J, Roberts PL, et al. Is the presence of medical trainees associated with increased mortality with weekend admission? BMC Med Educ 2014;14:4.

26. Shaheen AA, Kaplan GG, Myers RP. Weekend versus weekday admission and mortality from gastrointestinal hemorrhage caused by peptic ulcer disease. Clin Gastroenterol Hepatol 2009;7:303-10.

27. Ananthakrishnan AN, McGinley EL, Saeian K. Outcomes of weekend admissions for upper gastrointestinal hemorrhage: a nationwide analysis. Clin Gastroenterol Hepatol 2009;7:296-302e1.
28. Kostis WJ, Demissie K, Marcella SW, et al. Weekend versus weekday admission and mortality from myocardial infarction. N Engl J Med 2007;356:1099-109.

29. Kumar G, Deshmukh A, Sakhuja A, et al.; Milwaukee Initiative in Critical Care Outcomes Research (MICCOR) Group of Investigators. Acute myocardial infarction: a national analysis of the weekend effect over time. J Am Coll Cardiol 2015;65:217-8.

30. Aylin P, Bottle A, Majeed A. Use of administrative data or clinical databases as predictors of risk of death in hospital: comparison of models. BMJ 2007;334:1044.
Competing interests: Amit Garg has received research grants from Astellas for projects outside the submitted work. No other competing interests were declared.

This article has been peer reviewed.

Affiliations: Departments of Surgery (Dubois, Vogt, Vinden) and of Epidemiology and Biostatistics (Dubois, Vogt, Garg), Schulich School of Medicine and Dentistry, Western University, London, Ont.; Institute for Clinical Evaluative Sciences (Vinden, Winick-Ng, McClure, Bell, Garg), Toronto, Ont.; Lilibeth Caberto Kidney Clinical Research Unit (Roshanov), London Health Sciences Centre, London, Ont.; Department of Clinical Epidemiology and Biostatistics (Roshanov), McMaster University, Hamilton, Ont.; Department of Medicine (Bell), Mount Sinai Hospital, University of Toronto, Ont.

Contributors: All of the authors contributed to the conception and design of the study.
Jennifer Winick-Ng conducted the statistical analyses and had full access to the study data. Luc Dubois wrote the initial draft of the manuscript, and all of the authors critically revised the manuscript, approved the final version to be published and agreed to act as guarantors of the work.

Funding: This project was supported by a grant from the Academic Medical Organization of Southwestern Ontario (grant no. INN14-010).

Acknowledgements: This study was supported by the Institute for Clinical Evaluative Sciences (ICES) Western site. ICES is funded by an annual grant from the Ontario Ministry of Health and Long-Term Care. Core funding for ICES Western is provided by the Academic Medical Organization of Southwestern Ontario, the Schulich School of Medicine and Dentistry, Western University and the Lawson Health Research Institute. The opinions, results and conclusions reported in this article are those of the authors and are independent from the funding sources. No endorsement by the funding sources is intended or should be inferred. Parts of this material are based on data and information compiled and provided by the Canadian Institute for Health Information (CIHI); however, the analyses, conclusions, opinions and statements expressed herein are those of the authors and not necessarily those of CIHI. Amit Garg is supported by the Dr. Adam Linton Chair in Kidney Health Analytics.

Surgical Investigators Group at ICES Western: Luc Dubois, Sumit Dave, Eric Frechette, Sarah Jones, Andrew McClure, Danielle MacNeil, Jacob McGee, Richard Malthaner, Dave Nagpal, Stephen Pautler, Chris Vinden, Kelly Vogt, Blayne Welk, Jennifer Winick-Ng.

Accepted: June 24, 2016

Early release: Oct. 17, 2016

Correspondence to: Luc Dubois, Luc.Dubois@lhsc.on.ca 